\title{
Disability, Disablism, and COVID-19 Pandemic Triage
}

\author{
Jackie Leach Scully
}

Received: 22 April 2020 / Accepted: 27 July 2020

(C) Journal of Bioethical Inquiry Pty Ltd. 2020

\begin{abstract}
Pandemics such as COVID-19 place everyone at risk, but certain kinds of risk are differentially severe for groups already made vulnerable by pre-existing forms of social injustice and discrimination. For people with disability, persisting and ubiquitous disablism is played out in a variety of ways in clinical and public health contexts. This paper examines the impact of disablism on pandemic triage guidance for allocation of critical care. It identifies three underlying disablist assumptions about disability and health status, quality of life, and social utility, that unjustly and potentially catastrophically disadvantage people with disability in COVID-19 and other global health emergencies.
\end{abstract}

Keywords Disability · COVID-19 · Pandemic · Global health emergencies $\cdot$ Critical care $\cdot$ Rationing .

Allocation $\cdot$ Discrimination $\cdot$ Disablism

\section{Background}

In many countries affected by COVID-19 during early to mid-2020, healthcare planners and practitioners were or anticipated being confronted with an unfamiliar situation of extreme critical care rationing. A minority of the people infected by the SARS-CoV-2 virus become severely ill and eventually progress to needing intensive care including

J. L. Scully $(\bowtie)$

University of New South Wales, Sydney, NSW 2052, Australia

e-mail: jackie.leach.scully@unsw.edu.au mechanical ventilation, and demand for these resources has in some cases significantly exceeded supply. Later on, the same may apply to scarce antiviral treatment or vaccines as they become available. Decisions have to be made about which of the people who could potentially benefit get access to life-saving critical care if not all can. In any situation of pandemic triage, policymakers, the writers of guidelines, and frontline medical staff need guidance on how to make those decisions in the most humane and morally justifiable way, while patients, families, and the general public want to know the basis on which such decisions are being made.

During major health emergencies such as pandemics, resource allocation conventionally follows broadly utilitarian and egalitarian ethics. Although it would be an oversimplification to say that healthcare switches completely from a focus on individual patient interests to utilitarianism, there is a shift towards the public health logic of maximizing the good for the greatest number of people, while remaining committed to equality of treatment. The inevitable result is tension between imperatives, expectations, and customary practice.

In the early weeks and months of the COVID-19 pandemic a number of critical care guidelines and protocols were produced, primarily in countries with reasonably well-developed public health systems including Australia, the United States, several European countries, and the United Kingdom, with recommendations directed at various levels from national down to individual hospitals. Some focused on outlining fundamental ethical principles, others on providing practical decision trees or algorithms for frontline clinicians. Both types 
prompted concerned responses from representatives of people with disabilities and older people, as well as ethicists and practitioners, to the suggested criteria for excluding certain people from various stages of care. At worst, it was argued, these criteria discriminate unjustly against a broad class of people with disability or people over what was felt to be an arbitrary age limit (commonly 60 or 65). This brief paper focuses on people with disabilities, although much of it is applicable to older people as well.

One interpretation of this discrimination is a straightforward disvaluing of the lives of people with disability. In practice, it is more likely to be evidence of a complex (but no less unacceptable) form of disablism, defined as those practices of contemporary society that exclude, eradicate, and oppress people with sensory, physical, or intellectual impairments ${ }^{1}$. I suggest there are three overlapping but conceptually distinct disablist assumptions at play. Together, they critically endanger the rights of people with disability in a situation of pandemic triage. First, there are assumptions about the overall health status of people with disability; second, assumptions about their quality of life; and finally, assumptions about social utility, although these only become relevant where there is also confusion about the fundamental criteria that can justifiably be used in pandemic decision-making.

\section{Disablist Assumptions about Health}

The majority of pandemic triage protocols use probable clinical outcome as the primary decision-making criterion: that is, the likelihood that the treatment will save the life of a person who would otherwise not recover. Being able to recover without it, or being judged certain to die despite it, are both reasons for not receiving treatment. When there are many people who are equally in need of and also equally likely to benefit from a treatment that is in short supply, tie-breaker decisions could theoretically follow several different frameworks. Some authors have suggested that a lottery is the fairest procedure; others propose a first come, first served basis; others have argued that pandemic allocation

\footnotetext{
${ }^{1}$ Disablism is often discussed in conjunction with ableism. In disablism, the focus is on the exclusion of certain kinds of body; in ableism, focus is turned onto the kinds of body that society values and promotes. The disablist focus is more relevant to this paper's discussion. See Goodley 2014.
}

should deliberately try to compensate for historical discrimination against minority groups. The most frequent and, to many people, the most ethically justifiable move however is to continue along the path of best possible clinical outcome, by refining the clinical inclusion criteria in order to identify an increasingly narrow core of people most likely to benefit from treatment.

At this point the person's background health status becomes relevant, because some underlying conditions, including hypertension, cardiovascular disease, chronic obstructive pulmonary disease, and cancer, are already known to significantly decrease the chance of recovery from the severest form of COVID-19 (Wu and McGougan 2020). Crucially, some pandemic triage protocols appear to rely on the assumption that disability necessarily goes hand-in-hand with compromised health. In fact disability per se often has no overall health impact. In the absence of those conditions already known to reduce survival chances, people with sensory or mobility impairments, intellectual disability, or chronically disabling illnesses are as likely to benefit from treatment as anyone else. It is clear, however, that clinicians and policymakers who are unfamiliar with disability frequently conflate impairment that leads to disablement, with health status.

What makes the ethical terrain here more complicated is that some disabling conditions do involve health issues that are relevant to recovery from COVID-19. If someone already has damaged lungs, for example, or if long-term medication has led to seriously raised blood pressure, then the evidence so far available suggests that their chances of survival are lower. Nevertheless, the amount of individual variation means that global categorizations based purely on diagnostic labels can easily create injustice. For example, many of the people dying of COVID-19 are categorized as clinically obese, and associated morbidities such as hypertension probably contribute to their death; but not all individuals with a high BMI have seriously raised blood pressure or other relevant cardiovascular disorders (Dietz and Santos-Burgoa 2020; Lighter et al. 2020). Making the distinction between the general category and the particular individual is essential, particularly in the case of impairment, especially since bioethics and medical ethics both have dismal track records for oversimplifying the theoretical and experiential diversity hidden under the label of disability. 


\section{Disablist Assumptions about Quality of Life}

The second set of assumptions concerns the quality of life of disabled people. The consensus within pandemic ethics may be that critical care decisions should primarily be based on predicted clinical outcome, but the actual wording of decision trees and algorithms often fails to make clear whether the quality of life after survival is being taken into consideration as well. After all, in many healthcare decisions, it would be acceptable for predicted quality of life to play a role. Someone with terminal cancer and only a few months to live might decide to forego resuscitation after a future heart attack; or a person's prospects following a major stroke might be so poor that family and clinicians agree that continued life support is pointless.

But in these situations, the decisions are based on some knowledge of the details of individual lives and personal wishes. In an overwhelmingly disablist society, using predicted quality of life to allocate resources is likely to result in significant, and unjust, bias against people with disability. There is abundant empirical evidence of the wide discrepancy between non-disabled people's ideas about the quality of life with disability and disabled people's own evaluations (Albrecht and Devliger 1999; Ubel, Loewenstein and Jepson 2003). One reason for this "disability paradox" is the cognitive barrier that makes it hard to project oneself imaginatively into an unfamiliar kind of life without experiencing it as lesser instead of just different (Mackenzie and Scully 2007). If pandemic triage guidance does not explicitly state that quality-of-life considerations should be limited to extreme tie-breaker decisions, and in any case be based on evidence rather than assumption, it risks reinforcing the prejudice that a life with disability can never be as good - however a "good life" is defined — as a life without.

\section{Disablist Assumptions About Social Utility}

The third issue involves both disablism and a slippage of triage criteria. The slippage occurs in rationing scenarios when decision-makers start to reach beyond the prospect of clinical benefit to the individual and towards some idea of social utility, in other words how valuable that person will be to society if they are saved. Judged against this criterion, people with disability are then made more vulnerable by two further assumptions: first that social utility equates solely to economic productivity, and second that people with disability are economically unproductive and therefore a net burden to society. Both of these assumptions can be challenged.

A narrow form of social utility is acceptable to many people. It makes moral as well as practical sense, for instance, to prioritize the care of frontline medical staff, both because of the principle of reciprocity (since they have voluntarily put themselves at greater risk) and because it benefits everyone to get them back into action as rapidly as possible. In situations of more or less complete social collapse, there are further arguments for directing life-saving resources towards people who can offer a range of vital services other than healthcare. (Note that this would not necessarily discriminate against disability: a disabled person with experience in engineering or food production is much more socially useful than, say, a professor of bioethics.) But the SARS-CoV-2 pandemic is not complete social collapse, and there is no ethical justification for any slippage towards resource allocation by social utility in general or against people with disabilities in particular on the grounds of their assumed uselessness. To my knowledge, no COVID-19 pandemic triage guidance has explicitly made general social utility a basis for prioritizing critical care. However, it is not enough for guidance not to mention social utility criteria, since there is widespread public belief (and anxiety) that factors like social status, personal wealth, and ideas about "usefulness" do influence life or death decisions, while healthcare staff themselves may lapse into doing so under pressure. It's true that in private rather than state-supported healthcare systems personal wealth is not completely irrelevant to access to treatment, including critical care: the point is that official critical care guidance should explicitly rule out purely social factors as relevant to decision making.

\section{Discriminatory Norms}

Over the last half-century, disability scholarship has developed powerful analyses of the social, cultural, political, and economic forces that structure disablism in contemporary society. There is now an extensive literature demonstrating how our understanding of disability (what it is, what causes it, how we think we should respond to it) reflects culturally embedded ideas about normal human form, function, and behaviour: norms that are not often explicitly articulated or 
examined. In the COVID-19 pandemic these unexamined norms are being carried over into the reasoning of guidance on critical care and other areas. To give one example, on March 21, 2020 the U.K.'s National Institute for Health and Care Excellence (NICE) published its COVID-19 Rapid Guidance, which recommended prioritizing critical care resources based on a Clinical Frailty Scale (CFS) (NICE 2020). In its original form, the Rapid Guidance stated that the CFS should be used because it helps "to identify patients who are at increased risk of poor outcomes and who may not benefit from critical care interventions." However, as disabled people and representative organizations quickly pointed out, the CFS was designed for use in elderly people (Rockwood et al. 2005); it characterizes levels of frailty using criteria for instrumental and non-instrumental activities of daily living that may be irrelevant to the clinical outcome for COVID-19. The description of "frailty" at CFS level 5, for example, would apply to younger people with disability, including intellectual disabilities, cerebral palsy, and so on, who require help with some activities of daily living but are otherwise in good health and no less likely than other people of the same age to recover with treatment.

In response to these criticisms, the original NICE Guidelines were modified to acknowledge that the CFS is inappropriate for younger people with stable long-term disabilities and that any assessments should be individualized and holistic. The fact that similar clarifications have been issued when some other guidelines were challenged suggests that the problem is less one of conscious hostility towards disabled people and more lack of awareness that commonly accepted norms of form and function aren't necessarily universal-the unthinking acceptance of the kind of disablist assumptions outlined in this paper.

Some simple measures would help to ensure people with disability are not unjustly disadvantaged in pandemic critical care decisions:

- Pandemic critical care guidance should include not just the criteria for triage decisions but explain the reasoning behind them.

- Disability status should not be used as a simple proxy for health status.

- Critical care decisions should be based on knowledge of an individual's personal medical history, not on assumptions about background health status or quality of life (and preferably without relying solely on medical records, which are notoriously errorprone).

- Critical care decisions should scrupulously and specifically exclude considerations of broad social utility.

- Critical care guidance should state clearly that disabled people are to be treated as equally valuable and worthy of care as others.

Critical care is not the only area where people with disabilities are likely to be unfairly disadvantaged in a pandemic. The lack of thought given to their specific needs has already produced a situation in which people dependent on outside carers for basic functions essential to life, like eating and drinking, are told they must self-isolate, while residential care and nursing homes show disproportionately high rates of COVID-19 infections and death among their residents. These problems reflect an ongoing failure to consider people with disability as equal members of the community, with equal human and civil rights: a fundamental issue of disablism with potentially catastrophic consequences for disabled people in the COVID19 pandemic, and beyond.

\section{References}

Albrecht, G.L., and P.G. Devliger. 1999. The disability paradox: High quality of life against all odds. Social Science \& Medicine 48(8): 977-988.

Dietz, W., and C. Santos-Burgoa. 2020. Obesity and its implications for COVID-19 mortality. Obesity. ePub ahead of print, April 1. doi: https://doi.org/10.1002/oby.22818.

Goodley, D. 2014. Dis/ability studies: Theorising disablism and ableism. London, New York: Routledge.

Lighter, J., M. Phillips, S. Hochman, et al. 2020. Obesity in patients younger than 60 years is a risk factor for Covid-19 hospital admission. Clinical Infectious Diseases. ePub ahead of print, April 9. doi.org/https://doi.org/10.1093/cid/ciaa415.

Mackenzie, C., and J.L. Scully 2007. Moral imagination, disability and embodiment. Journal of Applied Philosophy 24(4): 335351.

National Institute for Health and Care Excellence. 2020. Covid-19 rapid guidance: Critical care in adults. NICE Guidance 159. https://www.nice.org.uk/guidance/ng159. Accessed April 20 , 2020.

Rockwood, K., X. Song, C. MacKnight, et al. 2005. A global clinical measure of fitness and frailty in elderly people. Canadian Medical Association Journal 173(5): 489-495. 
Ubel, P.A., G. Loewenstein, and C. Jepson. 2003. Whose quality of life? A commentary exploring discrepancies between health state evaluations of patients and the general public. Quality of Life Research 12(6): 599-607.

Wu, Z., and J. McGougan. 2020. Characteristics of and important lessons from the coronavirus disease 2019 (COVID-19) outbreak in China. Summary of a report of 72314 cases from the Chinese Center for Disease Control and Prevention. The
Journal of the American Medical Association 323(13): 1239-1242.

Publisher's note Springer Nature remains neutral with regard to jurisdictional claims in published maps and institutional affiliations. 